\title{
Existence result and conservativeness for a fractional order non-autonomous fragmentation dynamics
}

\author{
Emile Franc Doungmo Goufo ${ }^{a, *}$, Morgan Kamga Pene ${ }^{a}$, Jeanine N. Mwambakana ${ }^{b}$ \\ ${ }^{a}$ Department of Mathematical Sciences, University of South Africa, Florida Sciences Campus, 003, South Africa. \\ ${ }^{b}$ Department of Science, Mathematics and Technology Education, University of Pretoria, Pretoria, South Africa.
}

Communicated by A. Atangana

\begin{abstract}
We use the subordination principle together with an equivalent norm approach and semigroup perturbation theory to state and set conditions for a non-autonomous fragmentation system to be conservative. The model is generalized with the Caputo fractional order derivative and we assume that the renormalizable generators involved in the perturbation process are in the class of quasi-contractive semigroups, but not in the class $\mathcal{G}(1,0)$ as usually assumed. This, thenceforth, allows the use of admissibility with respect to the involved operators, Hermitian conjugate, Hille-Yosida's condition and the uniform boundedness to show that the operator sum is closable, its closure generates a propagator (evolution system) and, therefore, a $C_{0}$-semigroup, leading to the existence result and conservativeness of the fractional model. This work brings a contribution that may lead to the full characterization of the infinitesimal generator of a $C_{0}$-semigroup for fractional non-autonomous fragmentation and coagulation dynamics which remain unsolved. (c)2016 All rights reserved.
\end{abstract}

Keywords: Evolution system, propagator, semigroup perturbation, renormalization, fractional non-autonomous fragmentation, conservativeness.

2010 MSC: 26A33, 37N25, 49K40.

\section{Introduction and useful definitions}

The dynamical behavior of a system that can break up to produce smaller particles can be generalized to give the integro-differential system:

\footnotetext{
${ }^{*}$ Corresponding authors

Email address: dgoufef@unisa.ac.za (Emile Franc Doungmo Goufo)
} 


$$
\left\{\begin{aligned}
D_{t}^{\alpha} u(t, x) & =-a(t, x) u(t, x)+\int_{x}^{\infty} a(t, y) b(t, x, y) u(t, y) d y \\
u(\tau, x) & =u_{\tau}(x), 0 \leq \tau<t \leq T, x>0
\end{aligned}\right.
$$

where

$$
{ }_{0}^{C} D_{t}^{\alpha} u(x, t)=\frac{\partial^{\alpha}}{\partial t^{\alpha}} u(x, t)=\frac{1}{\Gamma(1-\alpha)} \int_{0}^{t}(t-r)^{-\alpha} \frac{\partial}{\partial r} u(x, r) d r
$$

with $0 \leq \alpha<1$ is the fractional derivative of $u(x, t)$ in the sense of Caputo [4], with $\Gamma$ the Gamma function

$$
\Gamma(z)=\int_{0}^{\infty} t^{z-1} e^{-1} d t, \quad z \in \mathbb{C} .
$$

For reasons of simplicity we denote ${ }_{0}^{C} D_{t}^{\alpha}=D_{t}^{\alpha}$. Moreover, $u$ is the particle mass distribution function $\left(u(\tau, x)=u_{\tau}(x)\right.$ is the mass distribution at some fixed time $\left.\tau \geq 0\right)$ with respect to the mass $x, b(t, x, y)$ is the distribution of particle masses $x$ spawned by the fragmentation of a particle of mass $y$, at the time $t \leq T \in \mathbb{R}$ and $a(t, x)$ is the evolutionary time-dependent fragmentation rate, that is, the rate at which mass $x$ particles break up at a time $t$. The first term on the right-hand side of (1.1) describes the reduction in the number of particles in the mass range $(x ; x+d x)$ due to the fragmentation of particles in the same range. The second term describes the increase in the number of particles in the range due to fragmentation of larger particles.

The idea here is to analyse the equation (1.1) in the Banach space $L_{1}\left(\mathfrak{I}, X_{1}\right)$ where $\mathfrak{I}=[0, T]$ and

$$
X_{1}=L_{1}([0, \infty), x d x)=\left\{\psi:\|\psi\|_{X_{1}}:=\int_{0}^{\infty} x|\psi(x)| d x<\infty\right\}
$$

using the theory of evolution semigroup.

Throughout the paper, we will consider the following regularity assumptions:

$$
\begin{aligned}
& (t, x) \rightarrow a(t, x) \in L_{1}\left(\left[0, T^{\prime}\right], L_{\infty}([k, l])\right) \text { for any } 0<k<l<\infty \text { and } T^{\prime} \in(0, T), \\
& b(t, x, y) \text { is a positive measurable function with } b(t, x, y)=0 \text { for all } x \geq y \text { and } 0 \leq t \leq T,
\end{aligned}
$$

and the local conservative law

$$
\int_{0}^{y} x b(t, x, y) d x=y
$$

for all $y \geq 0$ and $0 \leq t \leq T$.

Up to now, existence results and honesty have been proved for number of fragmentation (autonomous or non-local) models, see for e.g. [6, 9], where the authors used various methods including the substochastic semigroup theory. But models with time dependent coefficients (non-autonomous) remain barely touched and there are still only few papers devoted to their analysis (well-posedness, conservativeness, honesty, etc.) In [7], the authors used techniques of truncation to prove existence, uniqueness and mass conservation for a model of type (1.1). The authors in [13] use evolution semigroups approach which allows them to build on the substochastic semigroup theory and obtain an equivalent result as in [12]. In the analysis of the book by Tosio Kato [10] and later improved by Da Prato et al. [5], it is generally assumed that the generators $A(t)$ and $B(t)$ involved in the perturbation are of class $\mathcal{G}(1,0)$, but this condition is modified in this paper as we will see later in our analysis.

We begin by recasting (1.1) as the non-autonomous abstract Cauchy problem in $X_{1}$ :

$$
\left\{\begin{aligned}
D_{t}^{\alpha} u(t) & =Q(t) u(t), \quad 0 \leq \tau<t \leq T, \\
u(\tau) & =u_{\tau}
\end{aligned}\right.
$$

where $Q(t)$ is defined by $Q(t)=\mathcal{Q}(t)$ and represents the realization of $\mathcal{Q}(t)$ on the domain 


$$
D(Q(t))=\left\{u \in X_{1} ; \quad \mathcal{Q}(t) u(t) \in X_{1}\right\}
$$

with $(\mathcal{Q} u)$ defined as

$$
(\mathcal{Q} u)(t, x)=(\mathcal{Q} u)(t)(x)=-a(t, x) u(t, x)+\int_{x}^{\infty} a(t, y) b(t, x, y) u(t, y) d y,
$$

$\mathcal{Q}(t)$ is seen as the pointwise operation

$$
\psi(t, x) \longmapsto-a(t, x) \psi(t, x)+\int_{x}^{\infty} a(t, y) b(t, x, y) \psi(t, x) d y
$$

defined on the set of measurable functions. $\mathcal{Q}(t)$ indeed defines various operators. The aim here is to analyze the problem by rephrasing it in abstract form (abstract Cauchy problem (ACP)) as an ordinary differential equation.

Let us start with something simple and come back to the abstract Cauchy problem (1.4); To analyze and show the existence for this system, we will need a two-parameter family. We consider that for $0 \leq t \leq T$, $Q(t)$ is a bounded linear operator in $X_{1}$ and that $t \longrightarrow Q(t)$ is continuous in the uniform operator topology. We have the following definitions.

Definition 1.1 (Solution operator for a fractional model). Consider an operator $Q$ applying in the fractional model

$$
D_{t}^{\alpha}(u(x, t))=Q u(x, t), \quad 0<\alpha<1, x, t>0,
$$

subject to the initial condition

$$
u(x, 0)=f(x) \quad x>0,
$$

and defined in a Banach space $X_{1}$. A family $\left(G_{Q}(t)\right)_{t>0}$ of bounded operators on $X_{1}$ is called a solution operator of the fractional Cauchy problem (1.5) -1.6 if

(i) $G_{Q}(0)=I_{X_{1}}$;

(ii) $G_{Q}(t)$ is strongly continuous for every $t \geq 0$;

(iii) $Q G_{Q}(t) v=G_{Q}(t) Q v$ for all $v \in D(Q)$;

(iv) $G_{Q}(t) D(Q) \subset D(Q)$;

(v) $G_{Q}(t) v$ is a (classical) solution of the model (1.5)- 1.6 for all $v \in D(Q), t \geq 0$.

It is well-known $([3,6])$ that an operator $\widetilde{Q} \in \mathcal{G}(M, \omega)$ means $\widetilde{Q}$ generates a $C_{0}$-semigroup $\left(G_{\widetilde{Q}}(t)\right)_{t>0}$ so that there exists $M>0$ and $\omega$ such that

$$
\left\|G_{\widetilde{Q}}(t)\right\| \leq M e^{\omega t}
$$

Whence, by analogy if the fractional Cauchy problem 1.5 - 1.6$)$ has a solution operator $\left(G_{Q}(t)\right)_{t>0}$ verifying (1.7), then we say that $Q \in \mathcal{G}^{\alpha}(M, \omega)$. The solution operator $\left(G_{Q}(t)\right)_{t>0}$ is positive if

$$
G_{Q}(t) \geq 0
$$

and contractive if

$$
\left\|G_{Q}(t)\right\|_{X_{1}} \leq 1
$$

and we say $Q \in \mathcal{G}^{\alpha}(1,0)$.

Definition 1.2 (Evolution system [13] or propagator [11]). A two-parameter family of bounded linear operators $U(t, \tau), 0 \leq \tau<t \leq T$, is called propagator or evolution system if the following conditions are respected: 
(i) $U(\tau, \tau)=I$;

(ii) $U(t, r) U(r, \tau)=U(t, \tau)$ for $0 \leq \tau \leq r \leq t \leq T$;

(iii) $(t, \tau) \longrightarrow U(t, \tau)$ is strongly continuous for $0 \leq \tau \leq t \leq T$.

Next, we will find the propagator $U(t, \tau)$ associated with (1.4) such that $u(t)=U(t, \tau) u_{\tau}$ is in some sense a solution of (1.4) satisfying the initial condition $u(\tau)=u_{\tau}$. For that we need the following principle.

\subsection{Subordination principle [3, 6]}

Let us consider the order $\alpha$ as in (1.4). Subordination principle summarizes as follows: The same operator $Q$ guarantees better properties of the solution of (1.4) if we consider another order $\gamma$ such that $\gamma<\alpha$. In other words, the subordination principle states that if $Q$ generates a solution operator for the model (1.4) with the order $\alpha>0$, then it also generates a solution operator for the model (1.4) with any order $\gamma>0$ such that $\gamma<\alpha$. Hence, making use of this principle we just need to consider the model (1.4):

$$
\left\{\begin{aligned}
D_{t}^{\alpha} u(t) & =Q(t) u(t), 0 \leq \tau<t \leq T, \\
u(\tau) & =u_{\tau}
\end{aligned}\right.
$$

with $\alpha=1$.

Lemma 1.3. Let $Q(t)$ be a bounded linear operator in $X_{1}$ for $0 \leq t \leq T$. If the function $t \longrightarrow Q(t)$ is continuous in the uniform operator topology, then for every $u_{\tau} \in X_{1}$, the abstract Cauchy problem (1.8) has a unique classical solution u given by the relation:

$$
u(t)=u_{\tau}+\int_{\tau}^{t} Q(\varsigma) u(\varsigma) d \varsigma .
$$

Proof. See [13, Theorem 5.1, Chapter 5], the proof is done in a Banach space $X$ which is also true in $X_{1}$.

Theorem 1.4. There is a propagator $U(t, \tau)$ associated with the initial value problem (1.8) such that $U(t, \tau) u_{\tau}$ is its solution satisfying the initial condition $u(\tau)=u_{\tau}$.

Proof. From Lemma 1.3, we already have the existence and uniqueness of the solution. Let $u(t)$ be this solution. We define the so-called solution operator of $(1.8)$ by

$$
U(t, \tau) u_{\tau}=u(t) \text { for } 0 \leq \tau \leq t \leq T .
$$

- For every $u_{\tau} \in X_{1}, U(\tau, \tau) u_{\tau}=u(\tau)=u_{\tau}$ then $U(\tau, \tau)=I$ (condition (i)).

- For every $u_{\tau} \in X_{1}$, we have $U(t, \tau) u_{\tau}=u(t)$ and $U(t, r) U(r, \tau) u_{\tau}=U(t, r) u(r)=u(t)$, then condition (ii) follows from the uniqueness of the solution of (1.8).

- It is obvious that $U(t, \tau)$ is a linear operator defined in all $X_{1}$ since (1.8) is linear. The relation (1.9) implies $\|u(t)\| \leq\left\|u_{\tau}\right\|+\int_{\tau}^{t}\|Q(\varsigma)\|\|u(\varsigma)\| d \varsigma$ and from Gronwall's inequality we also have $\|u(t)\| \leq$ $\left\|u_{\tau}\right\| \exp \left(\int_{\tau}^{t}\|Q(\varsigma)\| d \varsigma\right)$. Then, 1.10 yields $\left\|U(t, \tau) u_{\tau}\right\|=\left\|u_{\tau}\right\| \exp \left(\int_{\tau}^{t}\|Q(\varsigma)\| d \varsigma\right)$, leading to

$$
\|U(t, \tau)\|=\exp \left(\int_{\tau}^{t}\|Q(\varsigma)\| d \varsigma\right)
$$

Whence, $U(t, \tau)$ is bounded and, therefore, strongly continuous. This concludes the proof.

The fact that $Q(t)$ is bounded actually makes this existence result easier to obtain. Unfortunately, $Q(t)$ is not always bounded and then, we use, in the following section, a different approach to obtain an equivalent result. 


\section{Equivalent norm approach}

Let us come back to the equation 1.8 and split it to have (1.1) written in the abstract form:

$$
\left\{\begin{aligned}
D_{t}^{\alpha} u(t) & =A(t) u(t)+B(t) u(t), \quad 0 \leq \tau<t \leq T, \\
u(\tau) & =u_{\tau},
\end{aligned}\right.
$$

where $A(t)$ is defined as $A(t)=\mathcal{A}(t)$ and represents the realization of $\mathcal{A}(t)$ on the domain $D(A(t))=\{u \in$ $\left.X_{1} ; \mathcal{A}(t) u \in X_{1}\right\}$, with

$$
\left[\mathcal{A}(t) u_{\tau}\right](x)=-a(t, x) u_{\tau}(x)
$$

and $B(t)$ is defined as $B(t)=\mathcal{B}(t)$ and represents the realization of $\mathcal{B}(t)$ on the domain $D(B(t))$ with

$$
\left[\mathcal{B}(t) u_{\tau}\right](x)=\int_{x}^{\infty} a(t, y) b(t, x, y) u_{\tau}(x) d y .
$$

Making use of the assumptions (1.2) and (1.3), $A(t)$ is bounded (then the previous theorem and lemma apply) and it is easy to show that (see [6] or [9]) for any $u \in X_{1}, B(t) u \in X_{1}$, so we can take $D(B(t))=D(A(t))$ and $(A(t)+B(t), D(A(t)))$ is well-posed operator. Let us put

$$
\mathcal{X}_{1}=L_{1}\left(\mathfrak{I}, X_{1}\right):=\left\{\psi:[0, T] \times \mathbb{R} \ni(\sigma, x) \rightarrow u(\sigma, x), \quad\|\psi\|_{1}:=\int_{0}^{T} \int_{0}^{\infty} x|\psi(\sigma, x)| d \sigma d x<\infty\right\} .
$$

In the following section the subscript $t$ in $A_{t}$ means the operator $A$ depends on time $t$ but is defined in $\mathcal{X}_{1}$ instead of $X_{1}$. Our aim here is to set some conditions in $\mathcal{X}_{1}$ under which the operator sum $K_{t}$ :

$$
K_{t} \psi=A_{t} \psi+B_{t} \psi, \text { on } D\left(A_{t}\right) \cap D\left(B_{t}\right)=D\left(A_{t}\right)
$$

is closable, its closure generates a propagator, and therefore is a $C_{0}$ semigroup. We rely on the following theorem which was originally proved by Tosio Kato [10] and later improved by Da Prato et al. [5].

Theorem 2.1. Consider in $\mathcal{X}_{1}$ the operators $A_{t}$ and $B_{t}$ be generators both belonging to the class $\mathcal{G}(1,0)$. If $D\left(A_{t}\right) \cap D\left(B_{t}\right)$ is dense in $\mathcal{X}_{1}$ and $\operatorname{ran}\left(A_{t}+B_{t}+\xi\right)$ is dense in $\mathcal{X}$ for some $\xi<0$, then $K_{t}$ is closable and its closure $\bar{K}_{t}$ is a generator from the class $\mathcal{G}(1,0)$.

The conditions $A_{t}$ and $B_{t} \in \mathcal{G}(1,0)$ are dropped in this paper to provide stronger results. Let us treat the integral operator in 2.1) as a perturbation of the much easier operator of multiplication by $a$ on $X_{1}$

$$
\left[A(t) u_{\tau}\right](x)=-a(t, x) u_{\tau}(x) .
$$

Recall that (Theorem 1.4) $A(t)_{t \in \mathfrak{I}}(\mathfrak{I}=[0, T])$ is a family of generators of $C_{0}$-semigroups in $X_{1}$, then, for any fixed $t \in \mathfrak{I}=[0, T], A(t)$ generates a propagator $U(t, \tau), 0 \leq \tau<t \leq T$ and this propagator defines a $C_{0}$-semigroup $\left(S_{A_{t}}(s)\right)_{s \geq 0}$ in $\mathcal{X}_{1}$ by the relation

$$
\left[S_{A_{t}}(s) u_{\tau}\right](\sigma)=\chi_{\mathfrak{I}} U(\sigma, \sigma-s) u_{\tau}(\sigma-s)=\chi_{\mathfrak{I}} \exp \left(-\int_{\sigma-s}^{\sigma} a(\xi, .) d \xi\right) u_{\tau}(\sigma-s), \quad u_{\tau} \in \mathcal{X}_{1},
$$

where $\chi_{\mathfrak{I}}$ is the characteristic function of $\mathfrak{I}$ and $\sigma \in \mathfrak{I}$. Then, when we say $A$ is the generator of $C_{0^{-}}$ semigroups in $\mathcal{X}_{1}$, we mean $A$ generates a propagator which defines a $C_{0}$-semigroups in $\mathcal{X}_{1}$ satisfying the relation (2.2). In the following definition, we assume that $Y$ is a subspace of $\mathcal{X}_{1}$ which is closed with respect to the norm $\|\cdot\|_{Y}$, not necessarily in the norm $\|\cdot\|_{1}$ (hence $Y$ is itself a Banach space).

Definition 2.2. Let $S_{A_{t}}(s)_{s \geq 0}$ be a $C_{0}$-semigroup and $A_{t}$ its infinitesimal generator. A subspace $Y$ of $\mathcal{X}_{1}$ is called $A_{t}$-admissible if it is an invariant subspace of $S_{A_{t}}(s), s \geq 0$ i.e., $S_{A_{t}}(s) Y \subseteq Y$, and the restriction of $S_{A_{t}}(s)$ to $Y$ (i.e., $S_{\breve{A}_{t}}(s):=S_{A_{t}}(s)_{\mid Y}, s \geq 0$ ) is a $C_{0}$-semigroup in $Y$ (i.e., it is strongly continuous in the norm $\left.\|\cdot\|_{Y}\right)$. 
If $T: Y \longrightarrow \mathcal{X}_{1}$ is the embedding operator of $Y$ into $\mathcal{X}_{1}$, we have

$$
S_{A_{t}}(\alpha) T u=T S_{\check{A}_{t}}(\alpha) u, u \in Y,
$$

which gives

$$
A_{t} T u=T \check{A}_{t} u, u \in D\left(\check{A}_{t}\right)
$$

with

$$
D\left(\check{A}_{t}\right)=\left\{u \in D\left(A_{t}\right) \cap Y: A_{t} u \in Y\right\} .
$$

Recall that the adjoint $A_{t}^{*}$ of $A_{t}$ is a linear operator from $D\left(A_{t}^{*}\right) \subset \mathcal{X}_{1}^{*}$ into $\mathcal{X}_{1}^{*}$ (the dual of $\mathcal{X}_{1}$ ) and is defined as follows: $D\left(A_{t}^{*}\right)$ is the set of all elements $x^{*} \in \mathcal{X}_{1}^{*}$ for which there is a $y^{*} \in \mathcal{X}_{1}^{*}$ such that

$$
\left\langle x^{*}, A_{t} x\right\rangle=\left\langle y^{*}, x\right\rangle \text { for all } x \in D\left(A_{t}\right)
$$

and if $x^{*} \in D\left(A_{t}^{*}\right)$ then $y^{*}=A_{t}^{*} x^{*}$ where $y^{*}$ is the element of $\mathcal{X}_{1}^{*}$ satisfying (2.4). With the assumptions 1.2 and $(1.3)$, we can state the following lemma.

Lemma 2.3. Let $\check{A}_{t}$ and $\check{B}_{t}$ two operators defined by (2.3) and satisfying, for all $\lambda \in(0, \infty) \subset \rho\left(\check{A}_{t}\right)$ and $\kappa \in(0, \infty) \subset \rho\left(\check{B}_{t}\right)$

$$
\begin{gathered}
\left\|\left(\lambda I-\check{A}_{t}\right)^{-1}\right\|_{Y} \leq \frac{1}{\lambda}, \\
\left\|\left(\kappa I-\check{B}_{t}\right)^{-1}\right\|_{Y} \leq \frac{1}{\kappa},
\end{gathered}
$$

in the Banach space $Y$. If either $\check{A}_{t}^{*}$ or $\check{B}_{t}{ }^{*}$ is densely defined in $Y^{*}$, then for any $\eta<0$ we have the inequality:

$$
|\eta|\|v\|_{Y^{*}} \leq\left\|\check{A}_{t}^{*} v+\check{B}_{t}^{*} v+\eta v\right\|_{Y^{*}}, v \in D\left(\check{A}_{t}^{*}\right) \cap D\left(\check{B}_{t}^{*}\right) .
$$

Proof. We suppose that $D\left(\check{B}_{t}^{*}\right)$ is dense in $Y^{*}$ and define the sum

$$
\check{K}_{t, \varepsilon}:=\check{A}_{t} u+\check{B}_{t}\left(I+\varepsilon \check{B}_{t}\right)^{-1} u, u \in D(\check{K})=D\left(\check{A}_{t}\right), \quad \varepsilon<0 .
$$

It is obvious that $\check{K}_{t, \varepsilon}$ also satisfies the relations 2.5 or 2.6 since $\check{A}_{t}$ and $\check{B}_{t}$ do. Then the relation 2.5 yields

$$
\left\|\left(\lambda I-\check{K}_{t, \varepsilon}\right)^{-1} u\right\|_{Y} \leq\left\|\left(\lambda I-\check{K}_{t, \varepsilon}\right)^{-1}\right\|_{Y}\|u\|_{Y} \leq \frac{1}{\lambda}\|u\|_{Y}, \quad u \in Y, \lambda>0, \varepsilon<0,
$$

leading to

$$
\begin{aligned}
\|u\|_{Y} & \leq \frac{1}{\lambda}\left\|\left(\lambda I-\check{K}_{t, \varepsilon}\right) u\right\|_{Y}, \quad u \in Y, \lambda>0, \varepsilon<0 \\
& \leq \frac{1}{\lambda}\left\|\left(\check{K}_{t, \varepsilon}-\lambda I\right) u\right\|_{Y}, \quad u \in Y, \lambda>0, \varepsilon<0 \\
& \leq \frac{1}{|\eta|}\left\|\left(\check{K}_{t, \varepsilon}+\eta I\right) u\right\|_{Y}, \quad u \in Y, \varepsilon<0, \quad \text { where we have set }-\lambda=\eta<0
\end{aligned}
$$

or

$$
\left\|\left(\check{K}_{t, \varepsilon}+\eta I\right) u\right\|_{Y} \geq|\eta|\|u\|_{Y}, \quad u \in D\left(\check{K}_{t, \varepsilon}\right)=D\left(\check{A}_{t}\right), \varepsilon<0, \eta<0 .
$$

Immediate properties of Hermitian conjugate give

$$
\left\|\left(\check{K}_{t, \varepsilon}^{*}+\eta I\right) v\right\|_{Y^{*}} \geq|\eta|\|v\|_{Y^{*}}, \quad v \in D\left(\check{K}_{t, \varepsilon}^{*}\right)=D\left(\check{A}_{t}^{*}\right), \varepsilon<0, \eta<0
$$

and

$$
\check{K}^{*}{ }_{t, \varepsilon} v=\check{A}_{t}^{*} v+\check{B}_{t}^{*}\left(I+\varepsilon \check{B}_{t}^{*}\right)^{-1} v, \quad v \in D\left(\check{K}_{t, \varepsilon}^{*}\right)=D\left(\check{A}_{t}^{*}\right), \quad \varepsilon<0 .
$$


Since $\check{B}_{t}^{*}$ is densely defined in $Y^{*}$, we have

$$
\left(I+\varepsilon{\check{B_{t}^{*}}}^{*}\right)^{-1} \longrightarrow I \quad \text { as } \varepsilon \nearrow 0
$$

and then,

$$
\check{K}_{t, \varepsilon}^{*} v \longrightarrow \check{A}_{t}^{*} v+\check{B_{t}^{*}} v \quad \text { as } \varepsilon \nearrow 0 .
$$

Substituting the latter relation in 2.8 yields $(2.7)$.

The approach is the same if we consider that it is rather $\check{A}_{t}^{*}$ which is densely defined in $Y^{*}$.

Corollary 2.4. Let $A_{t}$ and $B_{t}$ be two closed and densely defined operators satisfying, for all $\lambda \in(0, \infty) \subset$ $\rho\left(A_{t}\right)$ and $\kappa \in(0, \infty) \subset \rho\left(B_{t}\right)$

$$
\begin{gathered}
\left\|\left(\lambda I-A_{t}\right)^{-1}\right\|_{1} \leq \frac{1}{\lambda}, \\
\left\|\left(\kappa I-B_{t}\right)^{-1}\right\|_{1} \leq \frac{1}{\kappa}
\end{gathered}
$$

on $\mathcal{X}_{1}$. Let $Y \hookrightarrow \mathcal{X}_{1}$ be admissible with respect to $A_{t}$ and $B_{t}$ and let the operator $B_{t}$ verify

$$
Y \subseteq D\left(B_{t}\right)
$$

We assume that the induced generators $\check{A}_{t}$ and $\check{B}_{t}$, given by $(2.3)$, are closed, densely defined and satisfy the relations (2.5), and (2.6), respectively. If $D\left(B_{t}^{*}\right)$ is dense in $\mathcal{X}_{1}^{*}$ then

$$
|\eta|\|v\|_{Y^{*}} \leq\left\|\check{A}_{t}^{*} v+\check{B}_{t}^{*} v+\eta v\right\|_{Y^{*}}, \quad v \in D\left(\check{A}_{t}^{*}\right) \cap T^{*} \mathcal{X}_{1}^{*}, \quad \eta<0,
$$

where $T: Y \longrightarrow \mathcal{X}_{1}$ is the embedding operator.

Proof. Let $v \in D\left(\check{A}_{t}^{*}\right) \cap T^{*} \mathcal{X}_{1}^{*}$, then there is $w \in \mathcal{X}_{1}^{*}$ such that $v=T^{*} w$. we also have $T^{*} \mathcal{X}_{1}^{*} \subset D\left(\check{B}_{t}{ }^{*}\right)$ thanks to the condition (2.10). Therefore, the relation 2.9) of the previous lemma is applied to $v=T^{*} w$ as:

$$
\check{K}^{*}{ }_{t, \varepsilon} T^{*} w=\check{A}_{t}^{*} T^{*} w+\check{B_{t}^{*}}\left(I+\varepsilon \check{B_{t}^{*}}\right)^{-1} T^{*} w, \quad \varepsilon<0 .
$$

Since $T$ is the embedding operator of $Y$ into $\mathcal{X}_{1}$, we have

$$
\check{K}^{*}{ }_{t, \varepsilon} T^{*} w=\check{A}_{t}^{*} T^{*} w+\check{B}_{t}^{*} T^{*}\left(I+\varepsilon B_{t}^{*}\right)^{-1} w, \quad \varepsilon<0,
$$

which is well-posed since the operator $B_{t} T: Y \rightarrow \mathcal{X}_{1}$ is bounded thanks to 2.10). Since $B_{t}^{*}$ is densely defined in $\mathcal{X}_{1}^{*}$, we have

$$
\left(I+\varepsilon B_{t}^{*}\right)^{-1} \longrightarrow I \quad \text { as } \varepsilon \nearrow 0
$$

and then,

$$
\check{K}_{t, \varepsilon}^{*} T^{*} w=\check{A}_{t}^{*} T^{*} w+\check{B}_{t}^{*} T^{*}\left(I+\varepsilon B_{t}^{*}\right)^{-1} w \longrightarrow \check{A}_{t}^{*} T^{*} w+\check{B}_{t}^{*} T^{*} w \quad \text { as } \quad \varepsilon \nearrow 0 .
$$

Substituting the latter relation in 2.8) with $v=T^{*} w$ yields (2.11).

Remark 2.5. It is in general possible to find in the Banach Space $\mathcal{X}_{1}$ a new norm $\|$.$\| , which is equivalent to$ its natural norm

$$
\|u\|_{1}:=\int_{0}^{T} \int_{0}^{\infty} x|u(\sigma, x)| d \sigma d x
$$

such that the operator $A_{t}$ becomes a generator of the contraction semigroups on $\mathcal{X}_{1}$.

Indeed, since $A_{t}$ is the generator of a $C_{0}$-semigroup, let us say $\left(S_{A_{t}}(s)\right)_{s \geq 0}$, there is $M>0$ and $\omega$ such that for all $s \geq 0, \|\left(S_{A_{t}}(s) \|_{1} \leq M \mathrm{e}^{\omega s}\right.$. We have

$$
\|\left(S_{A_{t}}(s) u\left\|_{1} \leq M \mathrm{e}^{\omega s}\right\| u \|_{1}, \quad \forall u \in \mathcal{X}_{1} \leq M_{A_{t}} \mathrm{e}^{\omega s} .\right.
$$

We set

$$
\|u\|=\left(M M_{A_{t}}\right)^{-1} \sup _{s \geq 0} \mathrm{e}^{-\omega s} \int_{0}^{T} \int_{0}^{\infty} x\left|S_{A_{t}}(s) u(\sigma, x)\right| d \sigma d x .
$$


Simple calculations show that

$$
\int_{0}^{T} \int_{0}^{\infty} x|u(\sigma, x)| d \sigma d x=\|u\|_{1} \leq M M_{A_{t}}\|u\| \leq M^{2} M_{A_{t}}\|u\|_{1}, \quad \forall u \in \mathcal{X}_{1},
$$

which proves that the norm $\|$.$\| is equivalent to \|u\|_{1}$. On the other hand, we have

$$
\begin{aligned}
& \left\|S_{A_{t}}(\varsigma) u\right\|=\left(M M_{A_{t}}\right)^{-1} \mathrm{e}^{\omega \varsigma} \sup _{s \geq 0} \mathrm{e}^{-\omega(s+\varsigma)} \int_{0}^{T} \int_{0}^{\infty} x \mid\left(S_{A_{t}}(s) S_{A_{t}}(\varsigma) u(\sigma, x) \mid d \sigma d x,\right. \\
& \left\|S_{A_{t}}(\varsigma) u\right\| \leq\left(M M_{A_{t}}\right)^{-1} \mathrm{e}^{\omega \varsigma} \sup _{s \geq 0} \mathrm{e}^{-\omega(s+\varsigma)} M M_{A_{t}} \mathrm{e}^{\omega \varsigma} \mathrm{e}^{\omega s},
\end{aligned}
$$

which gives

$$
\left\|S_{A_{t}}(\varsigma) u\right\| \leq \mathrm{e}^{\omega \varsigma} .
$$

This proves that the semigroup $S_{A_{t}}(s)_{s \geq 0}$ is in the class $\mathcal{G}(1, \omega)$ of quasi-contractive semigroups in the Banach space $\mathcal{X}_{1}$ equipped with the norm $\|$.$\| . Next we extend this result and characterize the existence of$ an equivalent norm in $\mathcal{X}_{1}$ for the pair of generators $\left\{A_{t}, B_{t}\right\}$.

Definition 2.6. Let $A_{t}$ and $B_{t}$ be the generators of $C_{0}$-semigroups $S_{A_{t}}(s)_{s \geq 0}$ and $S_{B_{t}}(s)_{s \geq 0}$ in $\mathcal{X}_{1}$. The pair $\left\{A_{t}, B_{t}\right\}$ is called renormalizable with constants $\omega$ and $\nu$ if for any sequences $\left\{\alpha_{k}\right\}_{k=1}^{N}, \alpha_{k} \geq 0$ and $\left\{\delta_{k}\right\}_{k=1}^{N}, \delta_{k} \geq 0, n \in \mathbb{N}$, one has

$$
\sup _{\substack{\alpha_{1} \geq 0, \ldots, \alpha_{n} \geq 0 \\ \delta_{1} \geq 0, \ldots, \delta_{n} \geq 0 \\ n \in \mathbb{N}}} \mathrm{e}^{-\omega \Sigma \alpha_{k}} \mathrm{e}^{-\nu \Sigma \delta_{k}}\left\|S_{A_{t}}\left(\alpha_{1}\right) S_{B_{t}}\left(\delta_{1}\right) \ldots S_{A_{t}}\left(\alpha_{n}\right) S_{B_{t}}\left(\delta_{n}\right) u\right\|_{1}<\infty
$$

for each $u \in \mathcal{X}_{1}$.

Lemma 2.7. Let $A_{t}$ and $B_{t}$ two generators of $C_{0}$-semigroups in $\mathcal{X}_{1}$ equipped with its natural norm

$$
\|u\|_{1}:=\int_{0}^{T} \int_{0}^{\infty} x|u(\sigma, x)| d \sigma d x
$$

The pair $\left\{A_{t}, B_{t}\right\}$ is renormalizable with constants $\omega$ and $\nu$ if and only if there is an equivalent norm $\|$. in $\mathcal{X}_{1}$ such that $A_{t}$ and $B_{t}$ are closed, densely defined and we have $(\omega, \infty) \subset \rho\left(A_{t}\right)$ and $(\nu, \infty) \subset \rho\left(B_{t}\right)$, so that for all $\lambda>\omega, \kappa>\nu$,

$$
\begin{gathered}
\left\|\left(\lambda I-A_{t}\right)^{-1}\right\| \leq \frac{1}{\lambda-\omega}, \\
\left\|\left(\kappa I-B_{t}\right)^{-1}\right\| \leq \frac{1}{\kappa-\nu},
\end{gathered}
$$

with

$$
\rho\left(A_{t}\right)=\left\{\lambda \in \mathbb{C}, \lambda I-A_{t}: D\left(A_{t}\right) \rightarrow \mathcal{X}_{1} \text { invertible }\right\}
$$

and

$$
\rho\left(B_{t}\right)=\left\{\lambda \in \mathbb{C}, \lambda I-B_{t}: D\left(A_{t}\right) \rightarrow \mathcal{X}_{1} \text { invertible }\right\}
$$

the resolvent sets of $A_{t}$ and $B_{t}$, respectively.

Proof. Let us suppose that there is an equivalent norm $\|$.$\| in \mathcal{X}_{1}$ such that $A_{t}$ and $B_{t}$ are closed, densely defined and satisfy the relations 2.12) and (2.13), then using Hille-Yosida's condition, there are $\omega$ and $\nu$ such that for all $\alpha, \delta \geq 0$,

$$
\left\|S_{A_{t}}(\alpha) u\right\| \leq\|u\| \mathrm{e}^{\omega \alpha},\left\|S_{B_{t}}(\delta) u\right\| \leq\|u\| \mathrm{e}^{\nu \delta}, \text { for all } u \in \mathcal{X}_{1} .
$$


Since $\|\cdot\|$ and $\|\cdot\|_{1}$ are equivalent, there are $M \geq 0$ and $N \geq 0$ such that

$$
\left\|S_{A_{t}}(\alpha) u\right\|_{1} \leq M\left\|S_{A_{t}}(\alpha) u\right\| \leq M \mathrm{e}^{\omega \alpha}
$$

and

$$
\left\|S_{B_{t}}(\delta) u\right\|_{1} \leq N\left\|S_{B_{t}}(\delta) u\right\| \leq N \mathrm{e}^{\nu \delta}
$$

leading to

$$
\mathrm{e}^{-\omega \alpha}\left\|S_{A_{t}}(\alpha) u\right\|_{1} \leq M_{A_{t}}<\infty, \quad \forall \alpha \geq 0
$$

and

$$
\mathrm{e}^{-\nu \delta}\left\|S_{B_{t}}(\delta) u\right\|_{1} \leq N_{B_{t}}<\infty, \quad \forall \delta \geq 0 \text { and } u \in \mathcal{X}_{1} .
$$

We see that for any sequences $\left\{\alpha_{k}\right\}_{k=1}^{N}, \alpha_{k} \geq 0$, and $\left\{\delta_{k}\right\}_{k=1}^{N}, \delta_{k} \geq 0, n \in \mathbb{N}$, one has

$$
\sup _{\substack{\alpha_{1} \geq 0, \ldots, \alpha_{n} \geq 0 \\ \delta_{1} \geq 0, \ldots, \delta_{n} \geq 0 \\ n \in \mathbb{N}}} \mathrm{e}^{-\omega \Sigma \alpha_{k}} \mathrm{e}^{-\nu \Sigma \delta_{k}}\left\|S_{A_{t}}\left(\alpha_{1}\right) S_{B_{t}}\left(\delta_{1}\right) \ldots S_{A_{t}}\left(\alpha_{n}\right) S_{B_{t}}\left(\delta_{n}\right) u\right\|_{1}<\infty
$$

and the pair $\left\{A_{t}, B_{t}\right\}$ is renormalizable with constants $\omega$ and $\nu$. Conversely, we consider the pair $\left\{A_{t}, B_{t}\right\}$ renormalizable with constants $\omega$ and $\nu$. Then,

$$
M:=\sup _{\substack{\alpha_{1} \geq 0, \ldots, \alpha_{n} \geq 0 \\ \delta_{1} \geq 0, \ldots, \delta_{n} \geq 0 \\ n \in \mathbb{N},\|u\|_{1} \leq 1}} \mathrm{e}^{-\omega \Sigma \alpha_{k}} \mathrm{e}^{-\nu \Sigma \delta_{k}} \int_{0}^{T} \int_{0}^{\infty} x\left|S_{A_{t}}\left(\alpha_{1}\right) S_{B_{t}}\left(\delta_{1}\right) \ldots S_{A_{t}}\left(\alpha_{n}\right) S_{B_{t}}\left(\delta_{n}\right) u(\sigma, x)\right| d \sigma d x<\infty .
$$

Now we use the uniform boundedness principle showed in [10] and define in $\mathcal{X}_{1}$ the norm:

$$
\|u\|:=M^{-2} \sup _{\substack{\alpha_{1} \geq 0, \ldots, \alpha_{n} \geq 0 \\ \delta_{1} \geq 0, \ldots, \delta_{n} \geq 0 \\ n \in \mathbb{N}}} \mathrm{e}^{-\omega \Sigma \alpha_{k}} \mathrm{e}^{-\nu \Sigma \delta_{k}} \int_{0}^{T} \int_{0}^{\infty} x\left|S_{A_{t}}\left(\alpha_{1}\right) S_{B_{t}}\left(\delta_{1}\right) \ldots S_{A_{t}}\left(\alpha_{n}\right) S_{B_{t}}\left(\delta_{n}\right) u(\sigma, x)\right| d \sigma d x .
$$

Using the fact that

$$
\int_{0}^{T} \int_{0}^{\infty} x\left|S_{A_{t}}\left(\alpha_{1}\right) S_{B_{t}}\left(\delta_{1}\right) \ldots S_{A_{t}}\left(\alpha_{n}\right) S_{B_{t}}\left(\delta_{n}\right) u(\sigma, x)\right| d \sigma d x \leq M \mathrm{e}^{\omega \Sigma \alpha_{k}} \mathrm{e}^{\nu \Sigma \delta_{k}} \int_{0}^{T} \int_{0}^{\infty} x|u(\sigma, x)| d \sigma d x,
$$

it is clear that

$$
\|u\| \leq M^{-2} M \int_{0}^{T} \int_{0}^{\infty} x|u(\sigma, x)| d \sigma d x \text { and } \int_{0}^{T} \int_{0}^{\infty} x|u(\sigma, x)| d \sigma d x \leq M^{2}\|u\| \text { for } u \in \mathcal{X}_{1} .
$$

Then,

$$
\|u\| \leq M^{-1}\|u\|_{1} \text { and }\|u\|_{1} \leq M^{2}\|u\| \text { for } u \in \mathcal{X}_{1} .
$$

Hence the norms $\|$.$\| and \|.\|_{1}$ are equivalent. Moreover (2.14), 2.15), and the fact that $A_{t} \in \mathcal{G}(M, \omega)$ also yield

$$
\left\|S_{A_{t}}(\varsigma) u\right\| \leq M^{-2} \sup _{\substack{\alpha_{1} \geq 0, \ldots, \alpha_{n} \geq 0 \\ \delta_{1} \geq 0, \ldots, \delta_{n} \geq 0 \\ n \in \mathbb{N}}} \mathrm{e}^{-\omega \Sigma \alpha_{k}} \mathrm{e}^{-\nu \Sigma \delta_{k}} \int_{0}^{T} \int_{0}^{\infty} x\left|S_{A_{t}}\left(\alpha_{1}\right) S_{B_{t}}\left(\delta_{1}\right) \ldots S_{A_{t}}\left(\alpha_{n}\right) S_{B_{t}}\left(\delta_{n}\right) S_{A_{t}}(\varsigma) u(\sigma, x)\right| d \sigma d x
$$




$$
\begin{aligned}
& \leq M^{-2} \sup _{\substack{\alpha_{1} \geq 0, \ldots, \alpha_{n} \geq 0 \\
\delta_{1} \geq 0, \ldots, \delta_{n} \geq 0 \\
n \in \mathbb{N}}} \mathrm{e}^{-\omega \Sigma \alpha_{k}} \mathrm{e}^{-\nu \Sigma \delta_{k}}\left\|S_{A_{t}}\left(\alpha_{1}\right) S_{B_{t}}\left(\delta_{1}\right) \ldots S_{A_{t}}\left(\alpha_{n}\right) S_{B_{t}}\left(\delta_{n}\right) S_{A_{t}}(\varsigma) u\right\|_{1} \\
& \leq M^{-2} \sup _{\substack{\alpha_{1} \geq 0, \ldots, \alpha_{n} \geq 0 \\
\delta_{1} \geq 0, \ldots, \delta_{n} \geq 0 \\
n \in \mathbb{N}}} \mathrm{e}^{-\omega \Sigma \alpha_{k}} \mathrm{e}^{-\nu \Sigma \delta_{k}}\left\|S_{A_{t}}\left(\alpha_{1}\right) S_{B_{t}}\left(\delta_{1}\right) \ldots S_{A_{t}}\left(\alpha_{n}\right) S_{B_{t}}\left(\delta_{n}\right)\right\|_{1}\left\|S_{A_{t}}(\varsigma) u\right\|_{1} \\
& \leq M^{-2} \sup _{\substack{\alpha_{1} \geq 0, \ldots, \alpha_{n} \geq 0 \\
\delta_{1} \geq 0, \ldots, \delta_{n} \geq 0 \\
n \in \mathbb{N}}} \mathrm{e}^{-\omega \Sigma \alpha_{k}} \mathrm{e}^{-\nu \Sigma \delta_{k}}\left\|S_{A_{t}}\left(\alpha_{1}\right) S_{B_{t}}\left(\delta_{1}\right) \ldots S_{A_{t}}\left(\alpha_{n}\right) S_{B_{t}}\left(\delta_{n}\right)\right\|_{1} M \mathrm{e}^{\omega \varsigma} \\
& \leq M^{-2} \mathrm{e}^{\omega \varsigma}\|u\|_{1} \\
& \leq \mathrm{e}^{\omega \varsigma}\|u\| .
\end{aligned}
$$

We have

$$
\left\|S_{A_{t}}(\varsigma) u\right\| \leq \mathrm{e}^{\omega \varsigma}\|u\|, u \in \mathcal{X}_{1}
$$

On the same way we get

$$
\left\|S_{B_{t}}(\varsigma) u\right\| \leq \mathrm{e}^{\nu \varsigma}\|u\|, u \in \mathcal{X}_{1}
$$

which means that the generators $A_{t} \in \mathcal{G}(1, \omega)$ and $B_{t} \in \mathcal{G}(1, \nu)$ in the Banach space $\mathcal{X}_{1}$ endowed with the norm $\|$.$\| . Hence, A_{t}$ and $B_{t}$ are closed, densely defined, and satisfy the relations (2.12) and (2.13) in $\left(\mathcal{X}_{1},\|\cdot\|\right)$.

Actually we have in hands all the essential elements allowing us to state the following perturbation theorem.

Theorem 2.8. Let $A_{t}$ and $B_{t}$ be a renormalizable pair of generators of $C_{0}$-semigroups on $\mathcal{X}_{1}$ and the induced generators $\check{A}_{t}$ and $\check{B}_{t}$ be closed, densely defined, and satisfy the relations (2.5) and (2.6), respectively. Further, let the Banach space $Y \hookrightarrow \mathcal{X}_{1}$ be admissible with respect to operators $A_{t}$ and $B_{t}$ so that $Y \subseteq D\left(B_{t}\right)$. If either $\check{A}_{t}^{*}$ or $\check{B}_{t}^{*}$ is densely defined in $Y^{*}$, or only $B_{t}^{*}$ is densely defined in $\mathcal{X}_{1}^{*}$, then the closure $\overline{K_{t}}$ of the operator sum $K_{t}$ :

$$
K_{t} \psi=A_{t} \psi+B_{t} \psi, \text { on } D\left(A_{t}\right) \cap D\left(B_{t}\right)=D\left(A_{t}\right)
$$

exists and $\overline{K_{t}}$ is the generator of a $C_{0}$-semigroup.

Proof. We just have to prove that the range of $\left(K_{t}+\eta\right)$ for some $\eta<0$ is dense in $\mathcal{X}_{1}$ and apply Theorem 2.1. Let $T$ be the embedding operator of Corollary 2.4. we have by Definition 2.2 that

$$
A_{t} T u=T \check{A}_{t} u, \text { for } u \in D\left(\check{A}_{t}\right)
$$

and

$$
B_{t} T u=T \check{B}_{t} u, \text { for } u \in D\left(\check{B}_{t}\right) .
$$

We also have $D\left(\check{B}_{t}^{*}\right) \supseteq T^{*} \mathcal{X}_{1}^{*}$ since $D\left(B_{t}\right) \supseteq Y$. Therefore $D\left(K_{t}\right)$ is dense in $\mathcal{X}_{1}$ and we obtain $D\left(K_{t}\right) \supseteq$ $T D\left(\check{A}_{t}\right)$ since $\check{A}_{t}$ is closed and densely defined in $Y$ which is itself densely embedded in $\mathcal{X}_{1}$.

Now let $v \in D\left(K_{t}^{*}\right) \subseteq \mathcal{X}_{1}^{*}$, then we obtain

$$
\left\langle K_{t} T u, v\right\rangle=\left\langle A_{t} T u, v\right\rangle+\left\langle B_{t} T u, v\right\rangle
$$

or

$$
\left\langle u, K_{t}^{*} T^{*} v\right\rangle=\left\langle T \check{A}_{t} u, v\right\rangle+\left\langle u, B_{t}^{*} T^{*} v\right\rangle .
$$

Then,

$$
\left\langle\check{A}_{t} u, T^{*} v\right\rangle=\left\langle u, K_{t}^{*} T^{*} v\right\rangle-\left\langle u, B_{t}^{*} T^{*} v\right\rangle=\left\langle u, A_{t}^{*} T^{*} v\right\rangle, \quad u \in D\left(\check{A}_{t}\right),
$$


which means $T^{*} v \in D\left(\check{A}_{t}^{*}\right)$ and, then, $T^{*} D\left(K_{t}^{*}\right) \subseteq D\left(\check{A}_{t}^{*}\right)$. Since $D\left(\check{B}_{t}^{*}\right) \supseteq T^{*} \mathcal{X}_{1}^{*}$, we have

$$
T^{*} D\left(K_{t}^{*}\right) \subseteq D\left(\check{A}_{t}^{*}\right) \cap D\left(\check{B}_{t}^{*}\right) .
$$

Assuming now by contradiction that $\operatorname{ran}\left(K_{t}+\eta\right)$ is not dense in $\mathcal{X}_{1}$ for some $\eta<0$, then there is $v \in \mathcal{X}_{1}^{*}$ such that

$$
\left\langle\left(K_{t}+\eta\right) u, v\right\rangle=0, \quad u \in D\left(K_{t}\right),
$$

which means

$$
v \in D\left(K_{t}^{*}\right) \text { and }\left(K_{t}^{*}+\eta\right) v=0 .
$$

Hence,

$$
T^{*} v \in D\left(\check{A}_{t}^{*}\right) \cap D\left(\check{B}_{t}^{*}\right), \text { since } T^{*} D\left(K_{t}^{*}\right) \subseteq D\left(\check{A}_{t}^{*}\right) \cap D\left(\check{B}_{t}^{*}\right) .
$$

If $B_{t}^{*}$ is densely defined in $\mathcal{X}_{1}^{*}$ then we apply Corollary 2.4 and find that $T^{*} v=0$.

If either $\breve{A}_{t}^{*}$ or $\check{B}_{t}^{*}$ is densely defined in $Y^{*}$, then we apply Lemma 2.3 to find that $T^{*} v=0$. Therefore, we obtain $v=0$, which is impossible. Hence, $\operatorname{ran}\left(K_{t}+\eta\right)$ is dense in $\mathcal{X}_{1}$ for all $\eta<0$. Because $A_{t}$ and $B_{t}$ are a renormalizable pair of generators of $C_{0}$-semigroups on $\mathcal{X}_{1}$, we can use Lemma 2.7 and Hille-Yosida theorem to say that $A_{t}$ and $B_{t}$ are of class $\mathcal{G}(1,0)$. Therefore the operator $K_{t}=A_{t}+B_{t}$ is closable and the relation

$$
|\eta|\|u\|_{1} \leq\left\|K_{t} u+\eta u\right\|_{1}, \quad u \in D\left(K_{t}\right), \eta<0,
$$

yields the existence of the closure $\overline{K_{t}}$ of $K_{t}$. Theorem 2.1 completes the proof.

Corollary 2.9. Let the operators $A(t)=A$ and $B(t)=B$, independent of $t$ and satisfying the conditions of Theorem 2.8, then the closure $\overline{K(t)}=\bar{K}$ given as

$$
\bar{K} \psi=\overline{A \psi+B \psi} \text {, on } D(A) \cap D(B)=D(A)
$$

exists and is the generator of a $C_{0}$-semigroup.

Proof. In concrete applications, $A(t), t \in \mathfrak{I}$ is often a measurable family of generators or generators belonging uniformly to the class $\mathcal{G}(M, \omega)$, for some constants $M$ and $\omega$, and since we are in one dimensional case, one can easily verify, as shown in [13], that in this case the induced multiplication operator $A$ is an anti-generator or generator in $L p\left(\mathfrak{I}, X_{1}\right)$, for some $p \in[1, \infty)$ with $\mathfrak{I} \subseteq \mathbb{R}_{+}$. This reduces the problem to find certain conditions for the operator sum

$$
K \psi=A \psi+B \psi, \text { on } D(A) \cap D(B)=D(A)
$$

to be closable and its closure generates a $C_{0}$-semigroup and Theorem 2.8 ends the proof.

Remark 2.10. By the relation (2.2), it follows that the closure of $A(t)+B(t)$ generates a propagator.

This allows us to consider the following conservativeness result.

\section{Theorem 2.11.}

(a) The $C_{0}$-semigroup $\left(S_{\bar{K}_{t}}(s)\right)_{s \geq 0}$ generated by $\bar{K}_{t}=\overline{A_{t}+B_{t}}$ is conservative if and only if the associated propagator $U(t, \tau), 0 \leq \tau<t \leq T$, is conservative.

(b) If the operators $A_{t}$ and $B_{t}$, satisfy the conditions of Theorem 2.8, then the model 2.1) is conservative.

Proof. (a) We make use of the relation (2.2) and properties of $U$ given in Definition 1.2. (b) The second part of the proof follows from $(a)$ and based on [7, Theorem 6.13].

Before concluding, it is important to add that alternative and similar analysis to this work can be done using the recently introduced definitions of fractional derivatives with and without singular kernel [1, 2, 8 ] and this may yield an analogue result. 


\subsection{Concluding remarks}

We have exploited the subordination principle, set conditions on the generators involved in the semigroup perturbation and used the renormalization method, which is different from the preceding ones, to analyze the fractional models of type (2.1). We dropped the class $\mathcal{G}(1,0)$ for the class $\mathcal{G}(1, \nu)$ of quasi-contractive semigroups in $\mathcal{X}_{1}=L_{1}([0, T] \times[0, \infty), x d \sigma d x)$, and showed some existence results and conservativeness for the fractional non-autonomous fragmentation model (2.1), therefore, giving a stronger result than [5, 10, where the model was autonomous and not generalized with coefficients independent of time. The result obtained here can lead to the full characterization of the infinitesimal generator for the fractional nonautonomous fragmentation model (2.1) and later for fractional non-autonomous fragmentation-coagulation or non-autonomous transport-fragmentation-coagulation models, which remain open problems.

\section{References}

[1] A. Atangana, D. Baleanu, New fractional derivatives with nonlocal and non-singular kernel: theory and application to heat transfer model, Thermal Sci., 20 (2016), 763-769. 2

[2] A. Atangana, I. Koca, Chaos in a simple nonlinear system with Atangana-Baleanu derivatives with fractional order, Chaos Solitons Fractals, 89 (2016), 447-454. 2

[3] E. G. Bazhlekova, Subordination principle for fractional evolution equations, Fract. Calc. Appl. Anal., 3 (2000), 213-230. 1, 1.1.

[4] M. Caputo, Linear models of dissipation whose $Q$ is almost frequency independent, II, Reprinted from Geophys. J. R. Astr. Soc., 13 (1967), 529-539, Fract. Calc. Appl. Anal., 11 (2008), 4-14. 1

[5] G. Da Prato, P. Grisvard, Sommes d'opérateurs linéaires et équations différentielles opérationnelles, (French) J. Math. Pures Appl., 54 (1975), 305-387. 1, 2, 2.1

[6] E. F. Doungmo Goufo, A mathematical analysis of fractional fragmentation dynamics with growth, J. Funct. Spaces, 2014 (2014), 7 pages. 1, 1, 1.1, 2

[7] E. F. Doungmo Goufo, Non-local and non-autonomous fragmentation-coagulation processes in moving media, Ph.D. thesis, North-West University, South Africa, (2014). 1 , 2

[8] E. F. Doungmo Goufo, Application of the Caputo-Fabrizio fractional derivative without singular kernel to Kortewegde VriesBurgers equation, Math. Model. Anal., 21 (2016), 188-198. 2

[9] E. F. Doungmo Goufo, S. C. Oukouomi Noutchie, Honesty in discrete, nonlocal and randomly position structured fragmentation model with unbounded rates, C. R. Math. Acad. Sci. Paris, 351 (2013), 753-759. 1, 2

[10] T. Kato, Perturbation theory for linear operators, Die Grundlehren der mathematischen Wissenschaften, SpringerVerlag New York, Inc., New York, (1966). 1, 2, 2, 2.1

[11] H. Neidhardt, On linear evolution equations, III, Hyperbolic case, Technical report, Prepr., Akad. Wiss. DDR, Inst. Math. p-MATH-05/82, Berlin, (1982). 1.2

[12] S. C. Oukouomi Noutchie, Coagulation-fragmentation dynamics in size and position structured population models, $\mathrm{PhD}$ thesis, UKZN, (2009). 1

[13] A. Pazy, Semigroups of linear operators and applications to partial differential equations, Applied Mathematical Sciences, Springer-Verlag, New York, (1983). 1, 1.2, 1.1, 2 